\title{
On the Koebe Quarter Theorem for certain polynomials
}

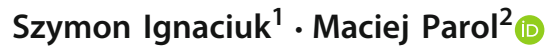

Received: 1 September 2019 / Revised: 29 January 2021 / Accepted: 6 February 2021 /

Published online: 24 February 2021

(C) The Author(s) 2021

\begin{abstract}
We study problems similar to the Koebe Quarter Theorem for close-to-convex polynomials with all zeros of derivative in $\mathbb{T}:=\{z \in \mathbb{C}:|z|=1\}$. We found minimal disc containing all images of $\mathbb{D}:=\{z \in \mathbb{C}:|z|<1\}$ and maximal disc contained in all images of $\mathbb{D}$ through polynomials of degree 3 and 4 . Moreover we determine the extremal functions for both problems.
\end{abstract}

Keywords Univalent polynomials $\cdot$ Koebe radius $\cdot$ Critical points

Mathematics Subject Classification $30 \mathrm{C} 10 \cdot 30 \mathrm{C} 15 \cdot 30 \mathrm{C} 25 \cdot 30 \mathrm{C} 45 \cdot 30 \mathrm{C} 75$

\section{Introduction}

Let $\mathcal{S}$ be the class of all analytic functions $f: \mathbb{D} \rightarrow \mathbb{C}$ normalized by $f(0)=$ $f^{\prime}(0)-1=0$ which are univalent. Let $\mathcal{H}_{d}$ be the class of all analytic functions $f: \mathbb{D} \rightarrow \mathbb{C}$ normalized by $f(0)=1$ and such that $f \neq 0$ in $\mathbb{D}$. For $\alpha, \beta \geq 0$ the Kaplan class $K(\alpha, \beta)$ is the set of all functions $f \in \mathcal{H}_{d}$ satisfying the condition

$$
\arg f\left(r \mathrm{e}^{\mathrm{i} \theta_{2}}\right)-\arg f\left(r \mathrm{e}^{\mathrm{i} \theta_{1}}\right) \leq \beta \pi-\frac{1}{2}(\alpha-\beta)\left(\theta_{1}-\theta_{2}\right)
$$

for $0<r<1$ and $\theta_{1}<\theta_{2}<\theta_{1}+2 \pi$ (see [9, pp. 32-33]). Kaplan clases were first defined by Sheil-Small in [10] and the restated version as in the condition (0.1) originally comes from [11]. The class $K(\alpha, \beta)$ is called the Kaplan class because using

\footnotetext{
$\triangle \quad$ Maciej Parol

mparol@kul.lublin.pl

Szymon Ignaciuk

szymon.ignaciuk@gmail.com

1 Department of Applied Mathematics and Computer Science, University of Life Sciences in Lublin, ul. Głęboka 28, 20-612 Lublin, Poland

2 Department of Mathematical Analysis, The John Paul II Catholic University of Lublin, ul. Konstantynów 1 H, 20-708 Lublin, Poland
} 
the Kaplan method [8], one can show that a normalized function $f$ analytic in $\mathbb{D}$ is close-to-convex of order $\alpha \geq 0$ if and only if $f^{\prime} \in K(\alpha, \alpha+2)$. Let $\mathcal{C}$ be the class of functions in $\mathcal{S}$ that are close-to-convex (see [4]). In particular $f \in \mathcal{C}$ if and only if $f^{\prime} \in K(1,3)$. The analytical properties and nice geometric interpretation make Kaplan classes find many applications to this day. In [7] Jahangiri and Ponnusamy use Kaplan classes both as a tool in assumptions as well as part of the result. In this article we will need the following theorem by Sheil-Small (see [12, p. 248], in [5] and [6] one can find additional information about polynomials with all zeros of derivative in $\mathbb{T}$ in the context of Kaplan classes).

Theorem A (Sheil-Small) Every polynomial of degree $n$ with all zeros on $\mathbb{T}$ belongs to $K(1,2 \pi / \lambda-n+1)$, where $\lambda$ is the minimal arclength between each pair of zeros.

Define $\mathbb{D}(r):=\{z \in \mathbb{C}:|z|<r\}$ for $r>0$,

$$
\mathrm{r}(\mathcal{F}):=\sup \left\{r>0: \mathbb{D}(r) \subset \bigcap_{f \in \mathcal{F}} f(\mathbb{D})\right\}
$$

and

$$
\mathrm{R}(\mathcal{F}):=\inf \left\{r>0: \bigcup_{f \in \mathcal{F}} f(\mathbb{D}) \subset \mathbb{D}(r)\right\}
$$

for any $\mathcal{F} \subset \mathcal{S}$. Bieberbach in 1916 proved the following theorem (see [1]).

Theorem B (Koebe Quarter Theorem) For every $f \in \mathcal{S}, \mathbb{D}(1 / 4) \subset f(\mathbb{D})$, i.e. $\mathrm{r}(\mathcal{S})=$ 1/4. Moreover the function

$$
\mathbb{D} \ni z \mapsto \frac{z}{(1-z)^{2}}
$$

is extremal for this inclusion.

The problem of determining $r(\mathcal{F})$ or $R(\mathcal{F})$ for any class $\mathcal{F} \subset \mathcal{S}$ is well known in the literature. Goodman devoted an entire chapter (see [4, pp. 113-120]) for so called Koebe domains. The concept of the Koebe domain for a set $\mathcal{F}$ is similar to the concept of $r(\mathcal{F})$ (usually they are equivalent). Directly from Theorem B we conclude that $r(\mathcal{F})=1 / 4$ for $\mathcal{F}$ being the class of starlike functions as well as the class of close-to-convex functions since the Koebe function is starlike and in the consequences close-to-convex. Hence, the Koebe domain for starlike functions and close-to-convex functions is a disc $\mathbb{D}(1 / 4)$.

Let $n \in \mathbb{N}$. Denote by $\mathcal{U}_{n}$ the class of all polynomials of degree $n$ belonging to $\mathcal{S}$. Additionally $\mathcal{U}_{n, \mathbb{R}}$ be the subclass of $\mathcal{U}_{n}$ containing only the polynomials with real coefficients. An interesting problem of determining $\mathrm{r}\left(\mathcal{U}_{n}\right)$ and $\mathrm{r}\left(\mathcal{U}_{n}, \mathbb{R}\right)$ was stated in [2]. It is easy to show that $\mathrm{r}\left(\mathcal{U}_{1, \mathbb{R}}\right)=\operatorname{r}\left(\mathcal{U}_{1}\right)=1$ and $\mathrm{r}\left(\mathcal{U}_{2, \mathbb{R}}\right)=\mathrm{r}\left(\mathcal{U}_{2}\right)=1 / 2$, but cases 
for $n \geq 3$ are far from trivial. Dmitrishin et al. [2] proved that

$$
\mathrm{r}\left(\mathcal{U}_{3, \mathbb{R}}\right)=\frac{3-\sqrt{5}}{2} \approx 0,382
$$

with the extremal function given by the formula

$$
\mathbb{D} \ni z \mapsto z+\frac{2 \sqrt{5}}{5} z^{2}+\frac{5-\sqrt{5}}{10} z^{3} \text {. }
$$

Moreover they proposed a class of functions which can be extremal at the problem of determining $\operatorname{r}\left(\mathcal{U}_{n, \mathbb{R}}\right)$ for $n \geq 4$.

Let $n \in \mathbb{N}$. Denote by $\overline{\mathcal{C}}_{n}(X)$ the class of all polynomials of degree $n$ belonging to $\mathcal{C}$ with all zeros of derivative on $X \subset \mathbb{C} \backslash \mathbb{D}$. Inspired by [2] and [3] we state the problem of determining $\mathrm{r}\left(\mathcal{C}_{n}(\mathbb{T})\right)$ and $\mathrm{R}\left(\mathcal{C}_{n}(\mathbb{T})\right)$. The classes $\mathcal{C}_{n}(\mathbb{T})$ are fairly large, $\mathcal{C}_{n}(\mathbb{T}) \subset \mathcal{U}_{n}$ and $\mathcal{C}_{n}(\mathbb{T}) \not \subset \mathcal{U}_{n, \mathbb{R}}$. Let us notice that cases $n=1$ and $n=2$ are trivial. It is easy to show that $\mathrm{r}\left(\mathcal{C}_{1}(\mathbb{T})\right)=\operatorname{R}\left(\mathcal{C}_{1}(\mathbb{T})\right)=1, \mathrm{r}\left(\mathcal{C}_{2}(\mathbb{T})\right)=1 / 2$ and $\mathrm{R}\left(\mathcal{C}_{2}(\mathbb{T})\right)=3 / 2$ with the extremal functions the same as in [2] for the problem of determining $\mathrm{r}\left(\mathcal{U}_{1}\right)$ and $\mathrm{r}\left(\mathcal{U}_{2}\right)$. In this article we determine $\mathrm{r}\left(\mathcal{C}_{n}(\mathbb{T})\right)$ and $\mathrm{R}\left(\mathcal{C}_{n}(\mathbb{T})\right)$ for $n=3$ and $n=4$.

\section{Main results}

In this section we solve the problem of determinig $\mathrm{r}\left(\mathcal{C}_{n}(\mathbb{T})\right)$ and $\mathrm{R}\left(\mathcal{C}_{n}(\mathbb{T})\right)$ for $n=3$ and $n=4$.

Theorem 1.1 The following equalities hold:

$$
\mathrm{r}\left(\mathcal{C}_{3}(\mathbb{T})\right)=\frac{\sqrt{10}}{6}
$$

and

$$
\mathrm{R}\left(\mathcal{C}_{3}(\mathbb{T})\right)=\frac{8+3 \sqrt{2}}{6}
$$

Moreover the function

$$
\mathbb{D} \ni z \mapsto z+\frac{-1+\mathrm{i}}{2} z^{2}-\frac{\mathrm{i}}{3} z^{3}
$$

is extremal for (1.1) and (1.2).

Proof Let $P \in \mathcal{C}_{3}(\mathbb{T})$. Then by Theorem A,

$$
\mathbb{D} \ni z \mapsto P(z):=\int_{0}^{z}\left(1-u \mathrm{e}^{-\mathrm{i} t_{1}}\right)\left(1-u \mathrm{e}^{-\mathrm{i} t_{2}}\right) \mathrm{d} u
$$


for certain $t_{1}, t_{2} \in \mathbb{R}$ such that $\pi / 2 \leq\left|t_{1}-t_{2}\right| \leq \pi$. Without loss of generality we can assume that $t_{1}:=0$ and $\pi / 2 \leq t_{2} \leq \pi$. Therefore

$$
\mathbb{D} \ni z \mapsto P(z):=z-\frac{1}{2}\left(1+\mathrm{e}^{-\mathrm{i} t_{2}}\right) z^{2}+\frac{1}{3} \mathrm{e}^{-\mathrm{i} t_{2}} z^{3}
$$

Since $P$ is a polynomial, so there exists the holomorphic extension of $P$ on $\mathbb{T}$. Setting $z:=\mathrm{e}^{\mathrm{i} \theta}$ for $\theta \in[0,2 \pi)$ we get

$$
\begin{aligned}
g(\theta) & :=\left|P\left(\mathrm{e}^{\mathrm{i} \theta}\right)\right|^{2}=\left|1-\frac{1}{2} \mathrm{e}^{\mathrm{i} \theta}-\frac{1}{2} \mathrm{e}^{\mathrm{i}\left(\theta-t_{2}\right)}+\frac{1}{3} \mathrm{e}^{\mathrm{i}\left(2 \theta-t_{2}\right)}\right|^{2} \\
& =\frac{29}{18}-\frac{4}{3} \cos (\theta)+\frac{1}{2} \cos \left(t_{2}\right)-\frac{4}{3} \cos \left(\theta-t_{2}\right)+\frac{2}{3} \cos \left(2 \theta-t_{2}\right)
\end{aligned}
$$

Therefore

$$
\begin{aligned}
g^{\prime}(\theta) & =\frac{4}{3}\left(\sin (\theta)+\sin \left(\theta-t_{2}\right)-\sin \left(2 \theta-t_{2}\right)\right) \\
& =\frac{16}{3} \sin \left(\frac{\theta}{2}\right) \sin \left(\frac{\theta-t_{2}}{2}\right) \sin \left(\frac{2 \theta-t_{2}}{2}\right)
\end{aligned}
$$

and as a consequence

$$
g^{\prime}(\theta)=0 \Longleftrightarrow \theta \in\left\{0, \frac{t_{2}}{2}, t_{2}, \frac{t_{2}+2 \pi}{2}\right\}
$$

Function $g$ has the minima at 0 and $t_{2}$ and the maxima at $t_{2} / 2$ and $t_{2} / 2+\pi$. Since

$$
g(0)=g\left(t_{2}\right)=\frac{5}{18}-\frac{1}{6} \cos \left(t_{2}\right) \in\left[\frac{5}{18}, \frac{4}{9}\right]
$$

and

$$
\begin{aligned}
g\left(\frac{t_{2}}{2}\right) & =\left(\frac{4}{3}-\cos \left(\frac{t_{2}}{2}\right)\right)^{2} \leq\left(\frac{4}{3}+\cos \left(\frac{t_{2}}{2}\right)\right)^{2} \\
& =g\left(\frac{t_{2}}{2}+\pi\right) \in\left[\frac{16}{9},\left(\frac{8+3 \sqrt{2}}{6}\right)^{2}\right]
\end{aligned}
$$

so for every $z \in \mathbb{T}$,

$$
\frac{\sqrt{10}}{6} \leq|P(z)| \leq \frac{8+3 \sqrt{2}}{6},
$$

which leads to (1.1) and (1.2). 
Now consider the function

$$
\mathbb{C} \ni z \mapsto f(z):=z+\frac{-1+\mathrm{i}}{2} z^{2}-\frac{\mathrm{i}}{3} z^{3} .
$$

Then $f_{\mid \mathbb{D}} \in \mathcal{C}_{3}(\mathbb{T})$,

$$
|f(1)|=\frac{\sqrt{10}}{6}
$$

and

$$
\left|f\left(\frac{-1-i}{\sqrt{2}}\right)\right|=\frac{8+3 \sqrt{2}}{6} .
$$

Fix $t_{2} \in[2 / 5 \pi, 2 / 3 \pi]$ and $t_{3} \in\left[2 t_{2}, \pi+t_{2} / 2\right]$. Define

$$
\begin{aligned}
& \Omega_{1}:=\left[\max \left\{0,-\frac{2}{3} \arccos \left(-\frac{6}{11}\right)+\frac{t_{2}+t_{3}}{3}\right\},-\frac{2}{3} \arccos \left(\frac{6}{11}\right)+\frac{t_{2}+t_{3}}{3}\right], \\
& \Omega_{2}:=\left[\frac{2}{3} \arccos \left(\frac{6}{11}\right)+\frac{t_{2}+t_{3}}{3}, \frac{2}{3} \arccos \left(-\frac{6}{11}\right)+\frac{t_{2}+t_{3}}{3}\right], \\
& \Omega_{3}:=\left[\frac{4}{3} \pi-\frac{2}{3} \arccos \left(-\frac{6}{11}\right)+\frac{t_{2}+t_{3}}{3}, \frac{4}{3} \pi-\frac{2}{3} \arccos \left(\frac{6}{11}\right)+\frac{t_{2}+t_{3}}{3}\right], \\
& \Omega_{4}:=\left[\frac{4}{3} \pi+\frac{2}{3} \arccos \left(\frac{6}{11}\right)+\frac{t_{2}+t_{3}}{3}, 2 \pi\right), \\
& {[0,2 \pi) \ni \theta \mapsto h_{1}(\theta):=\cos \left(\frac{3 \theta-t_{2}-t_{3}}{2}\right),} \\
& {[0,2 \pi) \ni \theta \mapsto h_{2}(\theta):=\cos \left(\frac{\theta}{2}\right) \cos \left(\frac{\theta-t_{2}}{2}\right) \cos \left(\frac{\theta-t_{3}}{2}\right),} \\
& {[0,2 \pi) \ni \theta \mapsto h(\theta):=\frac{11}{8} h_{1}(\theta)-h_{2}(\theta) .}
\end{aligned}
$$

Now we prove the following lemma.

Lemma 1.2 The function $h$ has exactly three zeros, one in each interval $\left(0, t_{2}\right),\left(t_{2}, t_{3}\right)$ and $\left(t_{3}, 2 \pi\right)$.

Proof Since

$$
\begin{gathered}
\left|\cos \left(\frac{\theta}{2}\right) \cos \left(\frac{\theta-t_{2}}{2}\right) \cos \left(\frac{\theta-t_{3}}{2}\right)\right| \leq\left|\cos \left(\frac{\theta}{2}\right) \cos \left(\frac{\theta-t_{3}}{2}\right)\right| \\
=\frac{1}{2}\left|\cos \left(\frac{2 \theta-t_{3}}{2}\right)+\cos \left(\frac{t_{3}}{2}\right)\right| \leq \frac{1}{2}\left(1+\left|\cos \left(\frac{t_{3}}{2}\right)\right|\right) \leq \frac{3}{4}
\end{gathered}
$$


for $\theta \in[0,2 \pi)$, so $h(\theta) \neq 0$ if

$$
\left|\cos \left(\frac{3 \theta-t_{2}-t_{3}}{2}\right)\right|>\frac{6}{11} .
$$

Therefore $h(\theta) \neq 0$ if $\theta \in[0,2 \pi) \backslash\left(\Omega_{1} \cup \Omega_{2} \cup \Omega_{3} \cup \Omega_{4}\right)$. For $\theta \in[0,2 \pi)$ we obtain

$$
h^{\prime}(\theta)=-\frac{25}{16} \sin \left(\frac{3 \theta-t_{2}-t_{3}}{2}\right)+\frac{1}{2} \sin \left(\frac{\theta}{2}\right) \sin \left(\frac{\theta-t_{2}}{2}\right) \sin \left(\frac{\theta-t_{3}}{2}\right) \text {. }
$$

Let us notice that

$$
\frac{3}{10} \pi<\arccos \left(\frac{6}{11}\right)<\frac{3}{8} \pi
$$

and

$$
\frac{3}{5} \pi<\arccos \left(-\frac{6}{11}\right)<\frac{3}{4} \pi
$$

Assume that $\theta \in \Omega_{1}$. Since $t_{2} \in[2 / 5 \pi, 2 / 3 \pi]$ and $t_{3} \in\left[2 t_{2}, \pi+t_{2} / 2\right]$, so by using (1.4) and (1.5) we get

$$
\begin{aligned}
& \frac{\theta}{2} \in\left[0, \frac{7}{30} \pi\right], \\
& \frac{\theta-t_{2}}{2} \in\left[-\frac{1}{4} \pi,-\frac{1}{30} \pi\right], \\
& \frac{\theta-t_{3}}{2} \in\left[-\frac{7}{12} \pi,-\frac{3}{10} \pi\right], \\
& \frac{3 \theta-t_{2}-t_{3}}{2} \in\left[-\frac{3}{4} \pi,-\frac{3}{10} \pi\right] .
\end{aligned}
$$

Therefore

$$
\begin{aligned}
& \sin \left(\frac{\theta-t_{2}}{2}\right), \sin \left(\frac{\theta-t_{3}}{2}\right), \sin \left(\frac{3 \theta-t_{2}-t_{3}}{2}\right)<0, \\
& \sin \left(\frac{\theta}{2}\right) \geq 0 .
\end{aligned}
$$

Hence $h^{\prime}(\theta)>0$ for $\theta \in \Omega_{1}$.

Assume that $\theta \in \Omega_{2}$. Since $t_{2} \in[2 / 5 \pi, 2 / 3 \pi]$ and $t_{3} \in\left[2 t_{2}, \pi+t_{2} / 2\right]$, so by using (1.4) and (1.5) we get

$$
\frac{\theta}{2} \in\left[\frac{3}{10} \pi, \frac{7}{12} \pi\right]
$$




$$
\begin{aligned}
& \frac{\theta-t_{2}}{2} \in\left[\frac{1}{10} \pi, \frac{19}{60} \pi\right], \\
& \frac{\theta-t_{3}}{2} \in\left[-\frac{7}{30} \pi, \frac{1}{20} \pi\right], \\
& \frac{3 \theta-t_{2}-t_{3}}{2} \in\left[\frac{3}{10} \pi, \frac{3}{4} \pi\right] .
\end{aligned}
$$

Therefore

$$
\begin{aligned}
& \sin \left(\frac{3 \theta-t_{2}-t_{3}}{2}\right) \in\left[\frac{\sqrt{2}}{2}, 1\right], \\
& \quad \sin \left(\frac{\theta}{2}\right) \sin \left(\frac{\theta-t_{3}}{2}\right) \in\left[\frac{1}{2}\left(\cos \left(\frac{2}{3} \pi\right)-1\right), \frac{1}{2}\left(\cos \left(\frac{2}{5} \pi\right)+1\right)\right] \\
& =\left[-\frac{3}{4}, \frac{3+\sqrt{5}}{8}\right], \\
& \quad \sin \left(\frac{\theta-t_{2}}{2}\right) \in\left(0, \sin \left(\frac{19}{60} \pi\right)\right] .
\end{aligned}
$$

Hence $h^{\prime}(\theta)<0$ for $\theta \in \Omega_{2}$.

Assume that $\theta \in \Omega_{3}$. Since $t_{2} \in[2 / 5 \pi, 2 / 3 \pi]$ and $t_{3} \in\left[2 t_{2}, \pi+t_{2} / 2\right]$, so by using (1.4) and (1.5) we get

$$
\begin{aligned}
& \frac{\theta}{2} \in\left[\frac{37}{60} \pi, \frac{9}{10} \pi\right], \\
& \frac{\theta-t_{2}}{2} \in\left[\frac{5}{12} \pi, \frac{19}{30} \pi\right], \\
& \frac{\theta-t_{3}}{2} \in\left[\frac{1}{12} \pi, \frac{11}{30} \pi\right], \\
& \frac{3 \theta-t_{2}-t_{3}}{2} \in\left[\frac{5}{4} \pi, \frac{17}{10} \pi\right] .
\end{aligned}
$$

Therefore

$$
\begin{aligned}
& \sin \left(\frac{3 \theta-t_{2}-t_{3}}{2}\right)<0, \\
& \sin \left(\frac{\theta}{2}\right), \sin \left(\frac{\theta-t_{2}}{2}\right), \sin \left(\frac{\theta-t_{3}}{2}\right)>0 .
\end{aligned}
$$

Hence $h^{\prime}(\theta)>0$ for $\theta \in \Omega_{3}$. 
Assume that $\theta \in \Omega_{4}$. Since $t_{2} \in[2 / 5 \pi, 2 / 3 \pi]$ and $t_{3} \in\left[2 t_{2}, \pi+t_{2} / 2\right]$, so by using (1.4) and (1.5) we get

$$
\begin{aligned}
& \frac{\theta}{2} \in\left[\frac{29}{30} \pi, \pi\right], \\
& \frac{\theta-t_{2}}{2} \in\left[\frac{23}{30} \pi, \frac{4}{5} \pi\right], \\
& \frac{\theta-t_{3}}{2} \in\left[\frac{13}{30} \pi, \frac{3}{5} \pi\right], \\
& \frac{3 \theta-t_{2}-t_{3}}{2} \in\left[\frac{23}{10} \pi, \frac{12}{5} \pi\right] .
\end{aligned}
$$

Therefore

$$
\begin{aligned}
& \sin \left(\frac{3 \theta-t_{2}-t_{3}}{2}\right) \in\left[\frac{1+\sqrt{5}}{4}, \sqrt{\frac{5+\sqrt{5}}{8}}\right], \\
& \sin \left(\frac{\theta}{2}\right) \sin \left(\frac{\theta-t_{3}}{2}\right) \in\left[\frac{1}{2}\left(\cos \left(\frac{2}{3} \pi\right)-1\right), \frac{1}{2}\left(\cos \left(\frac{2}{5} \pi\right)+1\right)\right] \\
& =\left[-\frac{3}{4}, \frac{3+\sqrt{5}}{8}\right], \\
& \quad \sin \left(\frac{\theta-t_{2}}{2}\right) \in\left[\sqrt{\frac{5-\sqrt{5}}{8}}, \sin \left(\frac{23}{30} \pi\right)\right] .
\end{aligned}
$$

Hence $h^{\prime}(\theta)<0$ for $\theta \in \Omega_{4}$.

Let us notice that

$$
\begin{aligned}
& h_{1}\left(-\frac{2}{3} \arccos \left(-\frac{6}{11}\right)+\frac{t_{2}+t_{3}}{3}\right)=h_{1}\left(\frac{2}{3} \arccos \left(-\frac{6}{11}\right)+\frac{t_{2}+t_{3}}{3}\right) \\
& =h_{1}\left(\frac{4}{3} \pi-\frac{2}{3} \arccos \left(-\frac{6}{11}\right)+\frac{t_{2}+t_{3}}{3}\right)=-\frac{6}{11}, \\
& h_{1}\left(-\frac{2}{3} \arccos \left(\frac{6}{11}\right)+\frac{t_{2}+t_{3}}{3}\right)=h_{1}\left(\frac{2}{3} \arccos \left(\frac{6}{11}\right)+\frac{t_{2}+t_{3}}{3}\right) \\
& =h_{1}\left(\frac{4}{3} \pi-\frac{2}{3} \arccos \left(\frac{6}{11}\right)+\frac{t_{2}+t_{3}}{3}\right) \\
& =h_{1}\left(\frac{4}{3} \pi+\frac{2}{3} \arccos \left(\frac{6}{11}\right)+\frac{t_{2}+t_{3}}{3}\right)=\frac{6}{11},
\end{aligned}
$$

Since $t_{2} \in[2 / 5 \pi, 2 / 3 \pi]$ and $t_{3} \in\left[2 t_{2}, \pi+t_{2} / 2\right]$, so $\Omega_{1} \subset\left[0, t_{2}\right), \Omega_{3} \cup \Omega_{4} \subset\left(t_{3}, 2 \pi\right)$ and $t_{2}<\min \left(\Omega_{2}\right)<t_{3}$. Since $t_{2} \notin \Omega_{1} \cup \Omega_{2} \cup \Omega_{3} \cup \Omega_{4}$, so by (1.3) we obtain 
$\left|h_{2}(\theta)\right|<3 / 4$ for $\theta \in \Omega_{1} \cup \Omega_{2} \cup \Omega_{3} \cup \Omega_{4}$. Therefore

$$
h\left(\min \left(\Omega_{1}\right)\right), h\left(\max \left(\Omega_{2}\right)\right), h\left(\min \left(\Omega_{3}\right)\right)<0
$$

and

$$
h\left(\max \left(\Omega_{1}\right)\right), h\left(\min \left(\Omega_{2}\right)\right), h\left(\max \left(\Omega_{3}\right)\right), h\left(\min \left(\Omega_{4}\right)\right)>0 .
$$

Moreover

$$
\begin{aligned}
h(0) & =\frac{7}{8} \cos \left(\frac{t_{2}+t_{3}}{2}\right)-\frac{1}{2} \cos \left(\frac{t_{3}-t_{2}}{2}\right)<0, \\
h\left(t_{3}\right) & =\frac{7}{8} \cos \left(\frac{2 t_{3}-t_{2}}{2}\right)-\frac{1}{2} \cos \left(\frac{t_{2}}{2}\right)<0, \\
h(2 \pi) & =-\frac{7}{8} \cos \left(\frac{t_{2}+t_{3}}{2}\right)+\frac{1}{2} \cos \left(\frac{t_{3}-t_{2}}{2}\right)>0 .
\end{aligned}
$$

Hence and by monotonicity of $h$ on intervals $\Omega_{1}, \Omega_{2}, \Omega_{3}$ and $\Omega_{4}$ we conclude that $h$ has exactly three zeros, one in each interval $\left(0, t_{2}\right),\left(t_{2}, t_{3}\right)$ and $\left(t_{3}, 2 \pi\right)$.

Using Lemma 1.2 we prove the following theorem.

Theorem 1.3 The following equalities hold:

$$
\mathrm{r}\left(\mathcal{C}_{4}(\mathbb{T})\right)=\frac{8-\sqrt{5}}{12}
$$

and

$$
\mathrm{R}\left(\mathcal{C}_{4}(\mathbb{T})\right)=\frac{20+5 \sqrt{5}}{12}
$$

Moreover the function

$$
\begin{aligned}
& \mathbb{D} \ni z \mapsto z-\frac{1-\mathrm{i} \sqrt{5+2 \sqrt{5}}}{4} z^{2}-\frac{3+\sqrt{5}+\mathrm{i} \sqrt{10+2 \sqrt{5}}}{12} z^{3} \\
& +\frac{1+\sqrt{5}-\mathrm{i} \sqrt{10-2 \sqrt{5}}}{16} z^{4}
\end{aligned}
$$

is extremal for (1.6) and (1.7).

Proof Let $P \in \mathcal{C}_{4}(\mathbb{T})$. Then by Theorem A,

$$
\mathbb{D} \ni z \mapsto P(z):=\int_{0}^{z}\left(1-u \mathrm{e}^{-\mathrm{i} t_{1}}\right)\left(1-u \mathrm{e}^{-\mathrm{i} t_{2}}\right)\left(1-u \mathrm{e}^{-\mathrm{i} t_{3}}\right) \mathrm{d} u
$$


for certain $t_{1}, t_{2}, t_{3} \in \mathbb{R}$ such that $2 \pi / 5 \leq\left|t_{1}-t_{2}\right| \leq\left|t_{2}-t_{3}\right| \leq 4 \pi / 5$. Without loss of generality we can assume that $t_{1}:=0,2 \pi / 5 \leq t_{2} \leq 2 \pi / 3$ and $2 t_{2} \leq t_{3} \leq t_{2} / 2+\pi$. Therefore $P$ is given by the formula

$$
\begin{aligned}
P(z):= & z-\frac{1}{2}\left(1+\mathrm{e}^{-\mathrm{i} t_{2}}+\mathrm{e}^{-\mathrm{i} t_{3}}\right) z^{2}+\frac{1}{3}\left(\mathrm{e}^{-\mathrm{i} t_{2}}+\mathrm{e}^{-\mathrm{i} t_{3}}\right. \\
& \left.+\mathrm{e}^{-\mathrm{i}\left(t_{2}+t_{3}\right)}\right) z^{3}-\frac{1}{4} \mathrm{e}^{-\mathrm{i}\left(t_{2}+t_{3}\right)} z^{4} .
\end{aligned}
$$

Since $P$ is a polynomial, so there exists the holomorphic extension of $P$ on $\mathbb{T}$. Setting $z:=\mathrm{e}^{\mathrm{i} \theta}$ for $\theta \in[0,2 \pi)$ we get

$$
\begin{aligned}
g(\theta):= & \left|P\left(\mathrm{e}^{\mathrm{i} \theta}\right)\right|^{2}=\frac{103}{48}+\frac{13}{18} \cos \left(t_{2}\right)+\frac{13}{18} \cos \left(t_{3}\right)+\frac{13}{18} \cos \left(t_{3}-t_{2}\right) \\
& -\frac{11}{6} \cos (\theta)-\frac{11}{6} \cos \left(\theta-t_{2}\right)-\frac{11}{6} \cos \left(\theta-t_{3}\right)-\frac{1}{3} \cos \left(\theta-t_{2}-t_{3}\right) \\
& -\frac{1}{3} \cos \left(\theta+t_{2}-t_{3}\right)-\frac{1}{3} \cos \left(\theta-t_{2}+t_{3}\right)+\frac{11}{12} \cos \left(2 \theta-t_{2}\right) \\
& +\frac{11}{12} \cos \left(2 \theta-t_{3}\right) \\
& +\frac{11}{12} \cos \left(2 \theta-t_{2}-t_{3}\right)-\frac{1}{2} \cos \left(3 \theta-t_{2}-t_{3}\right)
\end{aligned}
$$

Therefore

$$
g^{\prime}(\theta)=-\frac{32}{3} h(\theta) \sin \left(\frac{\theta}{2}\right) \sin \left(\frac{\theta-t_{2}}{2}\right) \sin \left(\frac{\theta-t_{3}}{2}\right) .
$$

The function

$$
[0,2 \pi) \ni \theta \mapsto \sin \left(\frac{\theta}{2}\right) \sin \left(\frac{\theta-t_{2}}{2}\right) \sin \left(\frac{\theta-t_{3}}{2}\right)
$$

has exactly three zeros at points $0, t_{2}$ and $t_{3}$. Hence by Lemma 1.2 we conclude that the function $g^{\prime}$ has exactly six zeros. Therefore $g$ has three minima at points $0, t_{2}$ and $t_{3}$ and three maxima one in each interval $\left(0, t_{2}\right),\left(t_{2}, t_{3}\right)$ and $\left(t_{3}, 2 \pi\right)$. Moreover

$$
\begin{aligned}
g(0)= & \frac{5}{16}-\frac{7}{36} \cos \left(t_{2}\right)-\frac{7}{36} \cos \left(t_{3}\right)+\frac{1}{18} \cos \left(t_{3}-t_{2}\right)+\frac{1}{12} \cos \left(t_{2}+t_{3}\right) \\
\geq & \frac{5}{16}-\frac{7}{36} \cos \left(\frac{2}{5} \pi\right)-\frac{7}{36} \cos \left(\frac{4}{5} \pi\right)+\frac{1}{18} \cos \left(\frac{4}{5} \pi\right) \\
& +\frac{1}{12} \cos \left(\frac{6}{5} \pi\right)=\frac{54-5 \sqrt{5}}{144}, \\
g\left(t_{3}\right)= & \frac{5}{16}+\frac{1}{18} \cos \left(t_{2}\right)-\frac{7}{36} \cos \left(t_{3}\right)-\frac{7}{36} \cos \left(t_{3}-t_{2}\right)+\frac{1}{12} \cos \left(2 t_{3}-t_{2}\right)
\end{aligned}
$$




$$
\begin{gathered}
\geq \frac{5}{16}+\frac{1}{18} \cos \left(\frac{4}{5} \pi\right)-\frac{7}{36} \cos \left(\frac{4}{5} \pi\right)-\frac{7}{36} \cos \left(\frac{2}{5} \pi\right) \\
+\frac{1}{12} \cos \left(\frac{6}{5} \pi\right)=\frac{54-5 \sqrt{5}}{144},
\end{gathered}
$$

and

$$
g\left(t_{2}\right)=\frac{5}{16}-\frac{7}{36} \cos \left(t_{2}\right)+\frac{1}{18} \cos \left(t_{3}\right)-\frac{7}{36} \cos \left(t_{3}-t_{2}\right)+\frac{1}{12} \cos \left(t_{3}-2 t_{2}\right) .
$$

Since $g\left(t_{2}\right)$ is increasing with respect to $t_{2}$ and $t_{3}$, so

$$
\begin{aligned}
g\left(t_{2}\right) & \geq \frac{5}{16}-\frac{7}{36} \cos \left(\frac{2}{5} \pi\right)+\frac{1}{18} \cos \left(\frac{4}{5} \pi\right)-\frac{7}{36} \cos \left(\frac{2}{5} \pi\right)+\frac{1}{12} \\
& =\left(\frac{8-\sqrt{5}}{12}\right)^{2}
\end{aligned}
$$

and the equality holds if and only if $t_{2}=2 / 5 \pi$ and $t_{3}=4 / 5 \pi$. This ends the proof of (1.6).

Now we prove (1.7). Let us notice that for $z \in \mathbb{D} \cup \mathbb{T}$,

$$
\begin{aligned}
|P(z)| & \leq \frac{5}{4}+\frac{1}{2}\left|1+\mathrm{e}^{-\mathrm{i} t_{2}}+\mathrm{e}^{-\mathrm{i} t_{3}}\right|+\frac{1}{3}\left|\mathrm{e}^{-\mathrm{i} t_{2}}+\mathrm{e}^{-\mathrm{i} t_{3}}+\mathrm{e}^{-\mathrm{i}\left(t_{2}+t_{3}\right)}\right| \\
& =\frac{5}{4}+\frac{5}{6}\left|1+\mathrm{e}^{\mathrm{i} t_{2}}+\mathrm{e}^{\mathrm{i} t_{3}}\right|=\frac{5}{4}+\frac{5}{6} \sqrt{3+2 \cos \left(t_{2}\right)+2 \cos \left(t_{3}\right)+2 \cos \left(t_{3}-t_{2}\right)} \\
& =\frac{5}{4}+\frac{5}{6} \sqrt{1+4 \cos ^{2}\left(\frac{t_{2}}{2}\right)+4 \cos \left(\frac{t_{2}}{2}\right) \cos \left(\frac{2 t_{3}-t_{2}}{2}\right)} \\
& \leq \frac{5}{4}+\frac{5}{6} \sqrt{1+4 \cos ^{2}\left(\frac{t_{2}}{2}\right)+4 \cos \left(\frac{t_{2}}{2}\right) \cos \left(\frac{3 t_{2}}{2}\right)}=\frac{5}{4}+\frac{5}{6}\left(1+2 \cos \left(t_{2}\right)\right) \\
& \leq \frac{5}{4}+\frac{5}{6}\left(1+\frac{\sqrt{5}-1}{2}\right)=\frac{20+5 \sqrt{5}}{12} .
\end{aligned}
$$

and the equality holds if and only if $z=\mathrm{e}^{\frac{7}{5} \pi \mathrm{i}}, t_{2}=2 / 5 \pi$ and $t_{3}=4 / 5 \pi$. This ends the proof of (1.7).

Moreover from the above considerations we conclude that the extremal function for (1.6) and (1.7) is

$$
\begin{aligned}
\mathbb{D} & \ni z \mapsto z-\frac{1+\mathrm{e}^{-\frac{2}{5} \pi \mathrm{i}}+\mathrm{e}^{-\frac{4}{5} \pi \mathrm{i}}}{2} z^{2}+\frac{\mathrm{e}^{-\frac{2}{5} \pi \mathrm{i}}+\mathrm{e}^{-\frac{4}{5} \pi \mathrm{i}}+\mathrm{e}^{-\frac{6}{5} \pi \mathrm{i}}}{3} z^{3}-\frac{\mathrm{e}^{-\frac{6}{5} \pi \mathrm{i}}}{4} z^{4} \\
& =z-\frac{1-\mathrm{i} \sqrt{5+2 \sqrt{5}}}{4} z^{2}-\frac{3+\sqrt{5}+\mathrm{i} \sqrt{10+2 \sqrt{5}}}{12} z^{3}
\end{aligned}
$$




$$
+\frac{1+\sqrt{5}-\mathrm{i} \sqrt{10-2 \sqrt{5}}}{16} z^{4}
$$

\section{Compliance with ethical standards}

Conflict of interest The authors have no relevant financial or non-financial interests to disclose. The authors have no conflicts of interest to declare that are relevant to the content of this article. All authors certify that they have no affiliations with or involvement in any organization or entity with any financial interest or non-financial interest in the subject matter or materials discussed in this manuscript. The authors have no financial or proprietary interests in any material discussed in this article.

Open Access This article is licensed under a Creative Commons Attribution 4.0 International License, which permits use, sharing, adaptation, distribution and reproduction in any medium or format, as long as you give appropriate credit to the original author(s) and the source, provide a link to the Creative Commons licence, and indicate if changes were made. The images or other third party material in this article are included in the article's Creative Commons licence, unless indicated otherwise in a credit line to the material. If material is not included in the article's Creative Commons licence and your intended use is not permitted by statutory regulation or exceeds the permitted use, you will need to obtain permission directly from the copyright holder. To view a copy of this licence, visit http://creativecommons.org/licenses/by/4.0/.

\section{References}

1. Bieberbach, L.: Über die Koeffizienten derjenigen Potenzreihen, welche eine schlichte Abbildung des Einheitskreises vermitteln, Sitzungsber. Preuss. Akad. Wiss. Phys-Math. Kl., pp. 940-955 (1916)

2. Dmitrishin, D., Dyakonov, K., Stokolos, A.: Univalent polynomials and Koebe's one-quarter theorem. Anal. Math. Phys. 9(3), 991-1004 (2019). https://doi.org/10.1007/s13324-019-00305-X

3. Dmitrishin, D., Smorodin, A., Stokolos, A.: Estimating the Koebe radius for polynomials. arXiv:1805.06927 (2018)

4. Goodman, A.W.: Univalent functions, vol. II. Mariner Pub. Co., Inc., Tampa, Florida (1983)

5. Ignaciuk, S., Parol, M.: Zeros of complex polynomials and Kaplan classes. Anal. Math. 46, 769-779 (2020). https://doi.org/10.1007/s10476-020-0044-8

6. Ignaciuk, S., Parol, M.: Kaplan classes of a certain family of functions. Ann. Univ. Mariae CurieSklodowska Sect. A Math. 74(2), 31-40 (2020). https://doi.org/10.17951/a.2020.74.2.31-40

7. Jahangiri, J.M., Ponnusamy, S.: Applications of subordination to functions with bounded boundary rotation. Arch. Math. 98, 173-182 (2012). https://doi.org/10.1007/s00013-012-0357-3

8. Kaplan, W.: Close-to-convex Schlicht functions. Michigan Math. J. 1, 169-185 (1952). https://doi.org/ $10.1307 / \mathrm{mmj} / 1028988895$

9. Ruscheweyh, S.: Convolutions in Geometric Function Theory. Seminaire de Math. Sup. 83, Les Presses de laUniversit'e de Montr'eal (1982)

10. Sheil-Small, T.: The Hadamard product and linear transformations of classes of analytic functions. J. Anal. Math. 34, 204-239 (1978). https://doi.org/10.1007/BF02790013

11. Sheil-Small, T.: Some remarks on Bazilevič functions. J. Anal. Math. 43, 1-11 (1983). https://doi.org/ 10.1007/BF02790175

12. Sheil-Small, T.: Complex Polynomials. Cambridge University Press, Cambridge (2002)

Publisher's Note Springer Nature remains neutral with regard to jurisdictional claims in published maps and institutional affiliations. 